\title{
Desarrollo de un Modelo Predictivo para la Calidad Superficial en un Proceso de Torneado sobre Aceros de Alta Resistencia
}

\author{
Development of Predictive Model for Surface Quality \\ in Turning Process for High Strength Steels
}

\author{
Lisandro Vargas Henríquez ${ }^{1}$, Cristian Pedraza Yapes², Carolina Devia Acosta ${ }^{3}$, Cindy Martínez Marrugo ${ }^{4}$ \\ ${ }^{1}$ M.Sc en Materiales y Procesos de Manufactura, Docente Programa de Mecánica, Grupo de Investigación IMTEF, lisandrovargas@mail. \\ uniatlantico.edu.co. ${ }^{2}$ M.Sc en Ingeniería Mecánica, Docente Programa de Mecánica., Ingeniera Mecánica, Universidad del Atlántico
}

Recibido 1/12/13, aceptado 3/03/2014

\section{RESUMEN}

La calidad superficial es uno de los indicadores de desempeño que tiene efecto sobre la aceptación o rechazo de un producto terminado. Un producto terminado que no cumpla con el acabado superficial requerido por el cliente es garantía de la generación de costos en el proceso de corte de metales manifestándose a través de grandes cantidades de desperdicio de material, re-trabajo de piezas, tiempos extras, etc.

El articulo presenta un estudio sobre la influencia de los parámetros de corte en la calidad superficial en el proceso de torneado cilíndrico de aceros. El trabajo experimental se ha desarrollado controlando el proceso de mecanizado y estableciendo valores fijos para los parámetros de Velocidad, Avance y Profundidad de corte. Se utiliza una técnica estadística basada en el análisis de varianza (ANOVA) y la Metodología de la Superficie de Respuesta para establecer el grado de dependencia del acabado final con los parámetros de corte y obtener un modelo que permite que la rugosidad superficial pueda predecirse mediante la combinación optima de los factores de estudio, logrando así que las especificaciones dimensionales proporcionales en el diseño de las piezas puedan cumplirse.

Palabras claves: Calidad Superficial, Parámetros de Corte, Torneado, Análisis de Varianza (ANOVA), Metodología de Superficie de Respuesta (RSM).

\section{ABSTRACT}

The surface quality is a performance indicator which has an effect on the acceptance or rejection of a finished product. If a finished product does not fulfill the final surface conditions established by the costumer it is consider as a guarantee for the costs in the cutting process of metals. Those costs are the results from big amounts of waste material, rework, and extra time, among others.

This article shows a study about the influence of cutting parameters in the surface quality regarding the steel cylindrical turning process. The experimental job has been developed due to the control in the machining process and establishing constants values for the speed, advance and depth of cut parameters. Statistics techniques like analysis of variance (ANOVA) and the response surface methodology are used in order to establish the dependence degree between final state and the cutting parameters. That relationship between those parameters is used for the construction of a model in order to predict the surface roughness and calculating the optimal combination of the factors under study, achieving in that way that the dimensional specifications during the pieces design can be fulfilled.

Keywords: Surface quality, Cutting Parameters, Turning Process, Analysis of Variance (ANOVA), Response Surface Methodology RSM 


\section{INTRODUCCIÓN}

Los principales problemas que encuentran los ingenieros en un proceso de mecanizado son: 1 . Determinar los valores óptimos de las variables que afectan el proceso para determinar una calidad deseada y 2. Maximizar el rendimiento de los sistemas de fabricación usando los recursos disponibles. Las decisiones que los ingenieros deben tomar están basadas en su experiencia considerando los fenómenos que se dan durante el proceso. En el campo del mecanizado, algunos de los citados fenómenos son altamente complejos e interactúan con un elevado número de parámetros. Para tener en cuenta este problema los investigadores proponen un modelo predictivo que intentan simular las condiciones durante el mecanizado y establecer una relación causa efecto entre diferentes factores y la calidad superficial del producto.

En los procesos de mecanizado el torneado representa una operación fundamental y concretamente el torneado de acabado una de las más comunes operaciones de arranque de viruta implicadas en una amplia variedad de industrias, donde la calidad es un factor muy importante en la producción. La Rugosidad Superficial y las tolerancias, son los parámetros críticos de mayor evaluación en el control de calidad del proceso productivo de muchas de las piezas mecanizadas. Los consumidores de estas piezas cada vez demandan un mejor acabado superficial y más estrechos márgenes de tolerancias, pero con menores costos, lo cual motiva hacia la investigación de los procesos que originan dicha calidad superficial para determinar la influencia de las variables involucradas en el acabado final.

La calidad superficial de una pieza se mide con la integridad superficial que a parte de la topología de la superficie, tiene en cuenta las propiedades mecánicas y metalúrgicas, muy importantes en la fatiga, resistencia a la corrosión o vida de servicio de la pieza. La topología de la superficie se identifica por su textura superficial, en la que se miden varias cantidades relacionadas con las desviaciones producidas en la pieza con respecto a la superficie nominal. La rugosidad superficial es el parámetro más representativo para describir la textura superficial debido a su influencia directa en la fricción, fatiga, resistencias electrónica y térmica. Es por esta razón la importancia del uso de modelos que permitan ayudar a determinar las condiciones óptimas de maquinado para la obtención de un buen acabado superficial. Benardos y Vosniakos [1], presentan en 2003 un estudio en el que clasifican 4 diferentes metodologías utilizadas por los investigadores hasta el momento para la predicción de la rugosidad superficial en el mecanizado. Una de esas metodologías es el diseño de experimentos, en la cual, técnicas como el diseño factorial, la metodología de superficies de respuesta y la regresión lineal múltiple se pueden desarrollar de forma combinada.
El paper muestra el estudio relevante acerca de la influencia de los parámetros de corte (avance, profundidad y velocidad de corte), y el material mecanizado en la rugosidad superficial de piezas obtenidas mediante los procesos de mecanizado de torneado, la importancia de investigar en esta área radica en el cada vez más creciente uso del mecanizado de alta velocidad en industrias como la automotriz y aeronáuticas que requieren acabados superficiales y tolerancias dimensionales de gran calidad.

\section{ESTADO DEL ARTE DE LA MODELIZACIÓN DEL ACABADO SUPERFICIAL}

El objetivo de las operaciones de mecanizado en la fase de acabado, es obtener la calidad superficial exigida (rugosidad superficial y tolerancia dimensional), con un desgaste mínimo de herramienta y con el mínimo tiempo posible, lo que repercute al final en la reducción de los costos de fabricación.

Según la documentación analizada, la caracterización del acabado superficial es compleja por la multitud de variables que pueden intervenir en el proceso de mecanizado, pero los resultados pueden aproximarse a partir de distintos métodos: Método de Taguchi, Diseño de Experimentos (DOE), Regresión lineal y análisis de coeficientes de correlación, Análisis geométrico, Algoritmos Genéticos (AG), análisis por Redes Neuronales Artificiales (RNA) o análisis Fractal.

Cada vez más, se intenta adaptar la metodología de experimentación existente para caracterizar o predecir el acabado superficial. En algunos casos exige una experimentación de grandes dimensiones, inviable para aplicarlo en el control de un proceso industrial concreto. Es necesario reducir al máximo el número de factores que pueden intervenir, conocer sus limitaciones, y entonces aplicar un método tal que con un mínimo de experiencias permita obtener una predicción bastante ajustada de la respuesta del proceso a controlar.

Las investigaciones iníciales sobre el tema se centraron en los factores que afectan a la rugosidad superficial. Boothroyd y Knight, en 1989 [2], dividió el estudio de la rugosidad en dos tipos: la rugosidad ideal obtenida debido a la geometría de la herramienta y al avance; y la rugosidad natural obtenida a partir de irregularidades del proceso de mecanizado como el desgaste de herramienta o la aparición de vibraciones.

En el 2001, Aboulatta [3], estableció un modelo de regresión lineal para la predicción de la rugosidad superficial basado en vibraciones de la máquina-herramienta en operaciones de torneado y los siguientes parámetros de corte: Velocidad de giro y avance, Profundidad de corte, Radio 
de punta, ángulo de posición y la relación entre longitud y diámetro de pieza. Los parámetros de rugosidad evaluados fueron $R a$, Rmáx y Rsk, a partir de la regresión lineal y análisis de los coeficientes de correlación. Como conclusión principal que su modelo dependiente de los parámetros de corte y vibraciones fue más exacto que el modelo sin considerar las vibraciones. Davim [4], establece una correlación entre la velocidad de corte, el avance y la profundidad, para obtener ecuaciones para $R a$ y $R t$, obtenidas por medio de regresión lineal múltiple.

En el 2004, Ghanij.A [5] evalúa los parámetros velocidad de corte, avance y profundidad para el fresado de acabado. El objetivo de los experimentos es poder optimizar los parámetros para obtener valores más bajos (mejores) de rugosidad y de fuerza de corte resultantes. Correa $\mathrm{M}$ et al [6], analiza los factores que afectan al acabado superficial de los procesos de mecanizado, con la mención de algunas de las técnicas de análisis y modelos predictivos elaborados por diversos grupos de investigación. Finalmente realizan la propuesta para un modelo de predicción de acabado superficial que forma parte de un sistema supervisor inteligente multiobjetivo. Para el modelo de estimación de rugosidad superficial, se propone utilizar métodos de regresión lineal a partir de modelos geométricos, métodos experimentales y/o técnicas de inteligencia artificial, como redes neuronales, lógica borrosa o sistemas neuroborrosos que podrían proporcionar mejores ajustes a salidas reales. Este modelo sería una propuesta innovadora para integrar aspectos de calidad superficial a un control en proceso que interactúe con controladores $\mathrm{CNC}$ de arquitectura abierta. Vivancos [7], presentan un trabajo cuyo objetivo es establecer las variables que deben ser consideradas en un modelo de rugosidad superficial en el mecanizado a alta velocidad, y para ello emplean el diseño de experimentos. Los parámetros considerados son velocidad de corte, avance y las profundidades axial y radial de corte, se toman como referencia dos modelos, que evalúan dos zonas diferentes de la pieza: una con el mecanizado en concordancia (downcutting) y otra con el mecanizado en oposición (upcutting). Vivancos [8], presenta un modelo matemático desarrollado por Diseño Experimental para determinar las condiciones óptimas de mecanizado para satisfacer las especificaciones de fabricación.

En el 2005, Vivancos [9], empleo un diseño de experimentos factorial fraccionado para determinar la influencia de la velocidad de corte, el avance, las profundidades axial y radial de corte, sobre la rugosidad en el fresado a alta velocidad.

\subsection{Base Conceptual}

Como indicador de la calidad superficial de una pieza se emplean por una parte los parámetros de rugosidad su- perficial y por otra parte los parámetros de tolerancia dimensional según sea el sector de fabricación.

En general, el perfil de rugosidad (Figura 1) y sus parámetros son los elementos característicos del estado superficial de una pieza y se encuentran bien definidos en la norma ISO 4287:1998 [10]. Según esta norma, para definir la calidad superficial de una pieza es necesario determinar una serie de parámetros obtenidos mediante un rugosímetro. Para realizar las mediciones de rugosidad, en general, se emplean rugosímetros de contacto mediante palpador, con filtro gaussiano. Los parámetros de ajuste básicos de estos dispositivos se encuentran definidos en la norma ISO 4288:1996 [11]. Las imperfecciones en la textura de la superficie no son consideradas en las normas citadas anteriormente, simplemente se indica, que los medidas deberían ser tomadas, allí donde no existan defectos provocados por otro proceso de fabricación. La norma ISO 8785:1998 [12] define las imperfecciones superficiales presentes en una pieza.

Figura 1. Perfil de Rugosidad y Superficie Ampliad [14]

Figure 1. Profile and Surface Roughness

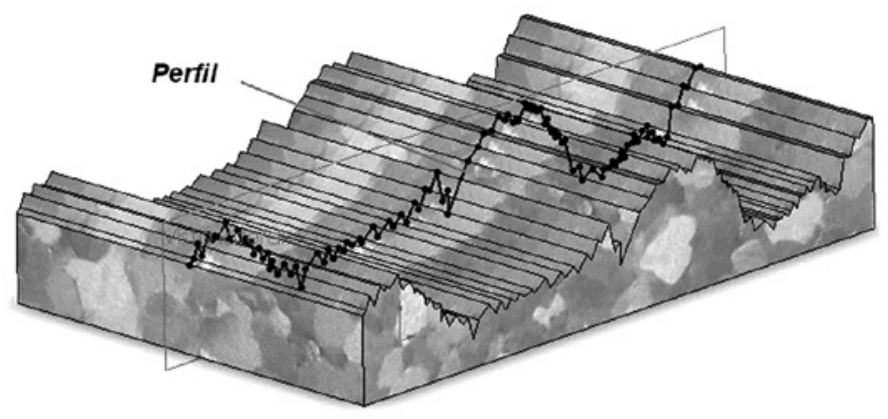

El parámetro de rugosidad, $R a$, es uno de los más utilizados y corresponde a la media aritmética de las desviaciones verticales del perfil con respecto a la línea media, dentro de una longitud de muestreo. En la ecuación 1 y Figura 2 queda reflejado el concepto del parámetro de rugosidad Ra.

$R a=\frac{1}{l} \int_{0}^{l}|Z(x)| d x \quad$ (Ec. 1)

El parámetro de rugosidad $R a$ mide las irregularidades verticales de la superficie a medir. Si existen irregularidades horizontales no las considera, por lo que es posible que superficies con distinto acabado superficial presenten el mismo valor de Ra, como se muestra en la Figura 3. 
Figura 2. Representación del Parámetro Ra[15]

Figure 2. Parameter Representation $R a$

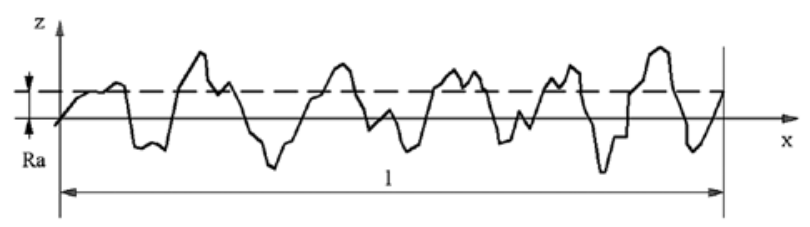

Figura 3. Distintas Superficies con Igual Parámetro Ra.[14] Figure 3. Different Surfaces with the same Parameter $R a$.
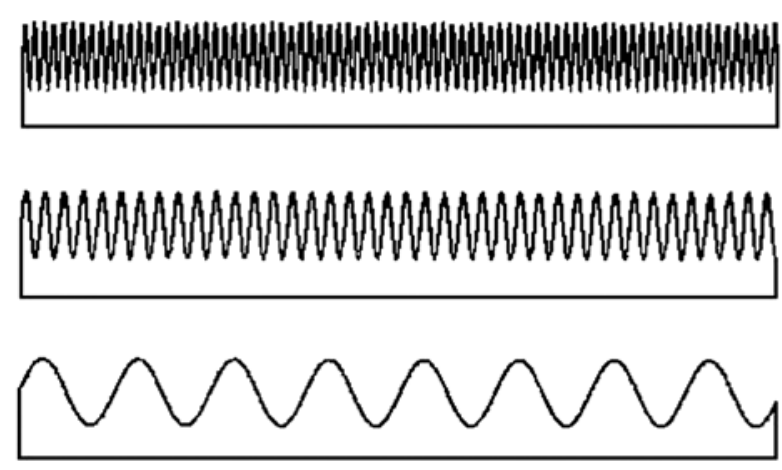

\section{ANÁLISIS DEL DISEÑO EXPERIMENTAL}

\subsection{Material y Procedimiento}

En esta etapa de la investigación se desea caracterizar la superficie de un acero AISI 4140 por el promedio de la rugosidad superficial $(\mathrm{Ra})$ reportada en micrómetros $(\mu \mathrm{m})$.

La investigación se lleva a cabo mediante las metodologías de análisis de varianza (ANOVA) y superficie de respuesta (RSM), utilizando 27 probetas de material acero bonificado AISI 4140 bajo las condiciones normales de suministro industrial. Las barras fueron provistas por la empresa BÖHLER. Las dimensiones iníciales de cada barra fueron en promedio de $25,4 \mathrm{~mm}$ de diámetro por $120 \mathrm{~mm}$ de largo. Las propiedades químicas y mecánicas del acero AISI 4140 se muestran en la tabla 1 y 2 respectivamente.

El acero 4140 es un acero aleado al cromo-molibdeno de buena penetración de temple y con buenas características de estabilidad en caliente hasta $400{ }^{\circ} \mathrm{C}$, sin fragilidad de revenido, muy versátil y apto para esfuerzos de fatiga y torsión. Puede ser endurecido superficialmente por temple directo (a la llama por inducción), obteniendo durezas de 57-62 Rockwell C. Se utiliza generalmente en estado bonificado a una resistencia a la tracción de $\left(88-100 \mathrm{~kg} / \mathrm{mm}^{2}\right)$ para: tornillería de alta resistencia grado 8 , ejes, bielas, engranajes, cigüeñales, cilindros de motores, rotores, arboles de turbinas de vapor, ejes traseros, etc. Muy utilizado en piezas forjadas como herramientas, llaves de mano, destornilladores. En la industria petrolera para taladros, barrenos tubulares, partes de bombas, vástagos de pistón, espárragos etc.

La operación de mecanizado seleccionada fue el cilindrado. El proceso de corte se realiza en un torno convencional horizontal marca IMO Turn Jumbo 66B-3000, una herramienta de corte de Tungsteno P-40-KEX $1 / 2$ ", Taladrina con lubricante y los siguientes parámetros de corte.

Tabla 3. Parámetros de Mecanizado

Table 3. Machining Parameters

\begin{tabular}{|c|c|c|c|}
\hline Nivel & $\begin{array}{c}\text { Avance }(\mathbf{m m} / \\
\text { rev) }\end{array}$ & $\begin{array}{c}\text { Profundidad } \\
(\mathbf{m m})\end{array}$ & $\begin{array}{c}\text { Velocidad de } \\
\text { Corte (RPM) }\end{array}$ \\
\hline 1 & 0,09 & 2 & 260 \\
\hline 2 & 0,15 & 3 & 330 \\
\hline 3 & 0,22 & 4 & 400 \\
\hline
\end{tabular}

Se procedió a cilindrar bajo estas especificaciones debido a su gran utilidad a nivel industrial, puesto que son condiciones bastante comunes. Se hicieron pruebas a todas

Tabla 1. Propiedades Química del Acero AISI 4140

Table 1. Chemical Properties of Steel AISI 4140

\begin{tabular}{|c|c|c|c|c|c|c|}
\hline $\begin{array}{c}\text { Composición } \\
\text { Química }\end{array}$ & \% C & \% Mn & \% P max & \% Cr & \% Mo & \% S max \\
\hline Análisis típico en \% & $0.38-0.43$ & $0.75-1.00$ & 0.035 & $0.8-1.1$ & $0.15-0.25$ & 0.04 \\
\hline
\end{tabular}

Tabla 2. Propiedades Mecánica del Acero AISI 4140

Table 2. Mechanical Properties of Steel AISI 4140

\begin{tabular}{|l|l|l|l|l|l|}
\hline $\begin{array}{c}\text { Estado de } \\
\text { Suministro }\end{array}$ & $\begin{array}{c}\text { Resistencia a la } \\
\text { Tracción, } \mathbf{k g} / \mathbf{m m}^{\mathbf{2}}\end{array}$ & $\begin{array}{c}\text { Limite Elástico, } \mathbf{k g} / \\
\mathbf{m m}^{\mathbf{2}}\end{array}$ & Alargamiento, \% & $\begin{array}{c}\text { Reducción de } \\
\text { Área, \% }\end{array}$ & \multicolumn{1}{c|}{ Dureza Brinell } \\
\hline Bonificado & $88-100$ & 75 & 16 & 50 & $260-320$ \\
\hline
\end{tabular}


las probetas, combinando entre sí los parámetros establecidos. Luego se tomaron cuatro medidas de la rugosidad superficial para cada probeta utilizando un rugosímetro marca Mahr Federal modelo POCKET SURF de contacto superficial. Cada medida se tomo en una longitud de barrido de $5 \mathrm{~mm}$, por lo tanto, la rugosidad de la superficie es un promedio de los valores tomados encima de la longitud de barrido. Las medidas tomadas se realizaron según la norma ASME B46.1-1995 para la textura de la superficie.

La figura 4, esquematiza la preparación del trabajo experimental para cada una de las probetas del estudio.

Figura 4. Variables que Influyen en el Acabado Superficial Durante el Torneado [16]

Figure 4. Variables Influencing Surface Finish During Turning

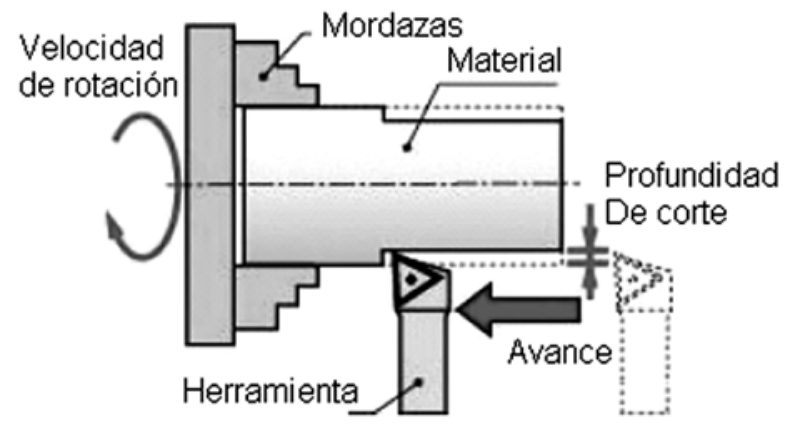

\subsection{Diseño de Experimentos: Ejecución y Resultados.}

A través de la metodología de Análisis de Varianza y Superficie de Respuesta y apoyado en el software Design Expert se generó un diseño factorial experimental aleatorio $3^{3}$, con cuatro replicas para cada una de las combinaciones. Con este arreglo experimental se generan 36 corridas experimentales para cada velocidad de corte empleada. En las tablas 4,5 y 6 , se muestran la combinación de los factores, parámetros de maquinado, a través de los tres niveles codificados, así como los datos de la variable respuesta rugosidad para las diferentes velocidades de corte (rpm).

Tabla 4. Valores Promedio Obtenidos de la Rugosidad a $260 \mathrm{rpm}$

Table 4. Returned Values Average Roughness to $260 \mathrm{rpm}$

\begin{tabular}{|c|c|c|c|c|c|c|c|}
\hline \multirow{2}{*}{\multicolumn{2}{|c|}{$\begin{array}{l}260 \\
\mathrm{rpm}\end{array}$}} & \multicolumn{6}{|c|}{ Avance } \\
\hline & & \multicolumn{2}{|c|}{0,09} & \multicolumn{2}{|c|}{0,15} & \multicolumn{2}{|c|}{0,22} \\
\hline \multirow{6}{*}{ 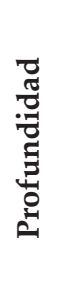 } & \multirow{2}{*}{2} & 4026 & 4103 & 2436 & 2423 & 6420 & 6452 \\
\hline & & 4436 & 4349 & 2410 & 2403 & 6426 & 6413 \\
\hline & \multirow{2}{*}{3} & 2462 & 3026 & 6410 & 6462 & 5436 & 5410 \\
\hline & & 3077 & 2718 & 6436 & 6513 & 5308 & 4949 \\
\hline & \multirow{2}{*}{4} & 1410 & 1590 & 2656 & 2667 & 5962 & 6282 \\
\hline & & 1744 & 1000 & 2692 & 3000 & 6256 & 5769 \\
\hline
\end{tabular}

Tabla 5. Valores Promedio Obtenidos de la Rugosidad a $330 \mathrm{rpm}$

Table 5. Returned Values Average Roughness to $330 \mathrm{rpm}$

\begin{tabular}{|c|c|c|c|c|c|c|c|}
\hline \multirow{2}{*}{\multicolumn{2}{|c|}{$\begin{array}{l}330 \\
\text { rpm }\end{array}$}} & \multicolumn{6}{|c|}{ Avance } \\
\hline & & \multicolumn{2}{|c|}{0,09} & \multicolumn{2}{|c|}{0,15} & \multicolumn{2}{|c|}{0,22} \\
\hline \multirow{6}{*}{ 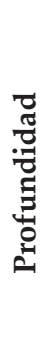 } & \multirow{2}{*}{2} & 5333 & 5256 & 1513 & 1769 & 1077 & 1231 \\
\hline & & 5205 & 5462 & 1692 & 1590 & 1467 & 1564 \\
\hline & \multirow{2}{*}{3} & 3000 & 3308 & 5667 & 5744 & 1256 & 1282 \\
\hline & & 3410 & 3179 & 6179 & 5974 & 1367 & 1308 \\
\hline & \multirow{2}{*}{4} & 2682 & 2513 & 2744 & 2744 & 974 & 795 \\
\hline & & 2769 & 3051 & 2923 & 3051 & 985 & 86 \\
\hline
\end{tabular}

Tabla 6. Valores Promedio Obtenidos de la Rugosidad a $400 \mathrm{rpm}$

Table 6. Returned Values Average Roughness to $330 \mathrm{rpm}$

\begin{tabular}{|c|c|c|c|c|c|c|c|}
\hline \multirow{2}{*}{\multicolumn{2}{|c|}{$\begin{array}{l}400 \\
\text { rpm }\end{array}$}} & \multicolumn{6}{|c|}{ Avance } \\
\hline & & \multicolumn{2}{|c|}{0,09} & \multicolumn{2}{|c|}{0,15} & \multicolumn{2}{|c|}{0,22} \\
\hline \multirow{6}{*}{ 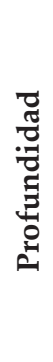 } & \multirow{2}{*}{2} & 2026 & 2308 & 2795 & 2923 & 5897 & 6154 \\
\hline & & 1897 & 1821 & 2723 & 2872 & 6410 & 6128 \\
\hline & \multirow{2}{*}{3} & 6430 & 6472 & 6451 & 6444 & 6425 & 6471 \\
\hline & & 6446 & 6413 & 6439 & 6533 & 6434 & 6423 \\
\hline & \multirow{2}{*}{4} & 6440 & 6432 & 6418 & 6442 & 5897 & 6410 \\
\hline & & 6416 & 6421 & 6456 & 6503 & 5974 & 6179 \\
\hline
\end{tabular}

En la tabla 7, se muestra el análisis de varianza donde se calcula y se analizan los efectos principales y sus interacciones.

Del análisis de varianza se concluye que existe una iteración significativa entre los parámetros velocidad de corte y el avance porque $\mathrm{F}_{0,05,4,27}=2.73$ y $\mathrm{F}_{0}>\mathrm{F}_{0,05,4,27}$. El valor del modelo $\mathrm{F}_{\mathrm{o}}$ de 83,03 implica que el modelo es significativo. Sólo hay una posibilidad de un $0,01 \%$ de que un "Valor F del Modelo" de esta magnitud pueda ocurrir debido a ruido. Además, también son significativos individualmente, el avance, la velocidad y la profundidad del corte, porque $\mathrm{F}_{0,05,24,27}=3,35$ y $\mathrm{F}_{0}>\mathrm{F}_{0,05,2,27}$. Es decir, que estos parámetros de corte afectan la rugosidad. Los valores de "Prob > F" menores de 0,0500 indican que los términos del modelo son significativos [13].

Los términos mayores de 0,1000 indican que los términos del modelo no son significativos, estos términos mejoran al reducir el modelo. 
Tabla 7. Análisis de Varianza para la Rugosidad Table 7. Analysis of Variance for Roughness

\begin{tabular}{|l|c|c|c|c|c|}
\hline $\begin{array}{c}\text { Fuente de } \\
\text { Variación }\end{array}$ & $\begin{array}{c}\text { Suma de } \\
\text { cuadrados }\end{array}$ & $\begin{array}{c}\text { Grados de } \\
\text { Libertad }\end{array}$ & $\begin{array}{c}\text { Media de } \\
\text { Cuadrados }\end{array}$ & Valor Fo & Prob $>$ F \\
\hline Modelo & $1,01 \mathrm{E}+0,8$ & 7 & 14370871 & 83,02831 & $<0,0001^{*}$ \\
\hline A - Velocidad & 4279275,1 & 1 & 4279275,1 & 24,72369 & $<0,0001$ \\
\hline B - Avance & 52762128 & 1 & 52762128 & 304,8354 & $<0,0001$ \\
\hline C - Profundidad & 8385,125 & 1 & 8385,125 & 0,048445 & 0,8274 \\
\hline AB & 5744355,1 & 1 & 5744355,1 & 33,18825 & $<0,0001$ \\
\hline AC & $2,07 \mathrm{E}+0,7$ & 1 & 20694961 & 119,566 & $<0,0001$ \\
\hline BC & 417241,13 & 1 & 417241,13 & 2,410628 & 0,1317 \\
\hline ABC & 16689753 & 1 & 16689753 & 96,4257 & $<0,0001$ \\
\hline Error & 1392722 & 27 & 51582,296 & & \\
\hline Total & 105442451 & 35 & & & \\
\hline
\end{tabular}

*Significante Fuente: Software Design Expert

Tabla 8. Estadística Descriptiva para los Datos

Table 8. Descriptive Statistics for Data

\begin{tabular}{|l|c|}
\hline \multicolumn{1}{|c|}{ Rugosidad } & Datos \\
\hline Desviación estándar & 416,03 \\
\hline $\mathrm{R}^{2}$ & 0,9425 \\
\hline $\mathrm{R}^{2}$ ajustado & 0,954 \\
\hline $\mathrm{R}^{2}$ predicho & 0,9506 \\
\hline Precisión Adecuada & 25,451 \\
\hline
\end{tabular}

Fuente: Software Design Expert

El valor $\mathrm{R}^{2}$ mide la proporción de la variabilidad total proporcionada por el modelo. La " $R^{2}$ predicha" de 0,9506 concuerda razonablemente con la " $\mathrm{R}^{2}$ ajustada" de 0,954 . La precisión adecuada mide la relación de la señal a ruido. Es deseable una relación mayor que 4. La relación de 25,451 indica una señal adecuada para usar este modelo para navegar el espacio del diseño.

La figura 5, de probabilidad normal de residuos muestra el diagnóstico del modelo. Los residuos son normalmente distribuidos y la suposición de igualdad de varianza no parece ser violada.
Figura 5. Probabilidad Normal de Residuales para los datos de Rugosidad

Figure 5. Normal probability of residuals for data Roughness

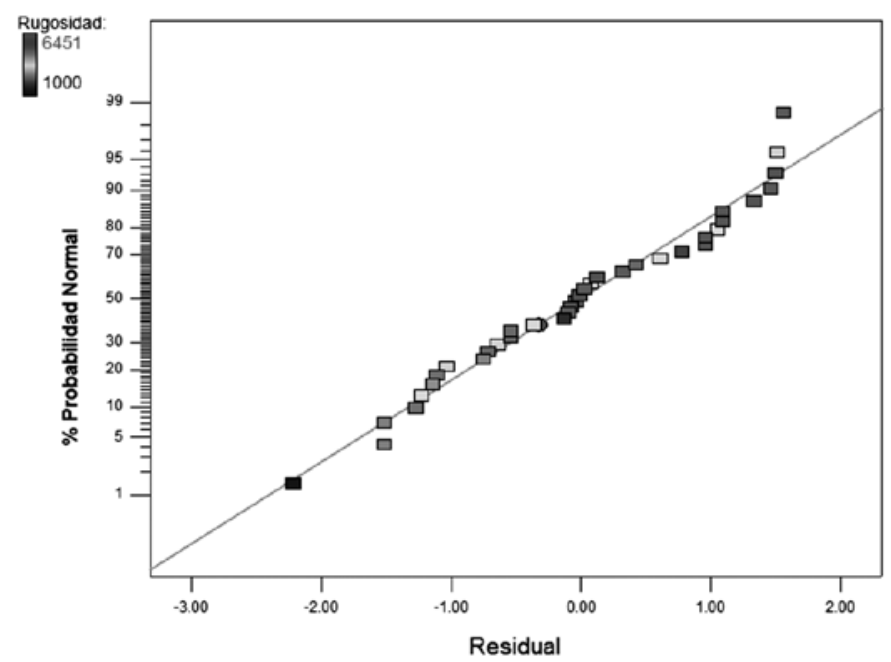

Fuente: Propia

La figura 6, corrobora la aceptación del modelo, debido a que los residuos no presentan una estructura, es decir no tienen un ordenamiento entre los datos. 
Figura 6. Residuales contra los Valores Ajustados de Rugosidad.

Figure 6. Adjusted residuals versus the roughness values

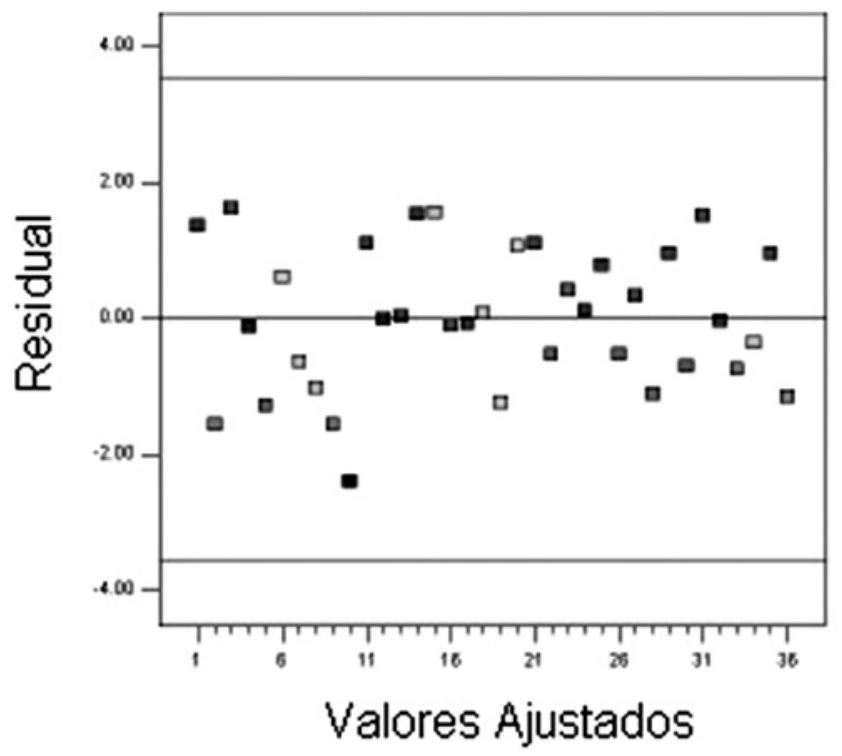

Fuente: Propia

Tabla 9. Resumen Estadístico del Modelo para la Rugosidad Tabla 9. Statistical Abstract Model for Roughness

\subsection{Análisis para Determinar la Superficie de Respuesta para la Rugosidad}

El anterior análisis fue un análisis cualitativo donde solo se podía predecir un nivel de los factores. Pero, lo que se busca es determinar el intervalo de los valores utilizados por medio de un modelo cuantitativo.

El modelo sugerido es un 2FI. El resumen estadístico del modelo se centra en los valores máximos del modelo de $\mathrm{R}^{2}$ ajustado y $\mathrm{R}^{2}$ predicho.

El nuevo análisis de varianza, es decir, la tabla ANOVA modificada, para la superficie de respuesta del modelo 2FI se muestra a continuación (Ver tabla 10).

El valor $\mathrm{F}$ del modelo de 22,82 implica que el modelo es significante. Hay sólo un $0.01 \%$ de posibilidad que este valor F ocurra debido a ruido. Los valores de "Prob > F" menos de 0.0500 indican que los términos del modelo son significantes. En este caso A, B, $\mathrm{A}^{*} \mathrm{~B}, \mathrm{~A}^{*} \mathrm{C}, \mathrm{A}^{2}$ del modelo son significantes. Los valores mayores de 0.01 indican que los términos del modelo no son significantes. Por tanto, no son significantes el termino $\mathrm{C}$ y $\mathrm{B}^{*} \mathrm{C}$.

\begin{tabular}{|l|c|c|c|c|c|l|}
\hline \multicolumn{1}{|c|}{ Fuente } & Desviación Estándar & $\mathbf{R}^{2}$ & $\mathbf{R}^{2}$ Ajustado & $\mathbf{R}^{2}$ Predicho & PRESS & \\
\hline Lineal & 1229,74 & 0,5411 & 0,498 & 0,4126 & $6,194 \mathrm{E}+07$ & \\
\hline$\underline{2 F I}$ & $\underline{861,76}$ & $\underline{0,7958}$ & $\underline{0,7535}$ & 0,6857 & $\underline{3,314 \mathrm{E}+07}$ & Sugerido \\
\hline Cuadrático & 803,62 & 0,8285 & 0,7856 & 0,7188 & $2,965 \mathrm{E}+07$ & Aliased \\
\hline
\end{tabular}

Fuente: Software Design Expert

Tabla 10. Tabla ANOVA del Modelo sugerido 2FI para la Rugosidad

Tabla 10. ANOVA Table for Model 2FI suggested Roughness

\begin{tabular}{|l|c|c|c|c|c|}
\hline Fuente de Variación & Suma de cuadrados & Grados de Libertad & Media de Cuadrados & Valor Fo & Prob > F \\
\hline Modelo & $8,736 \mathrm{E}+07$ & 5 & $1,670 \mathrm{E}+07$ & 22,82 & $<0,0001$ \\
\hline A - Velocidad & $4,279 \mathrm{E}+06$ & 1 & $4,279 \mathrm{E}+06$ & 5,85 & 0,0219 \\
\hline B - Avance & $5,276 \mathrm{E}+07$ & 1 & $5,270 \mathrm{E}+07$ & 72,1 & $<0,0001$ \\
\hline C - Profundidad & 8385,12 & 1 & 8385,12 & 0,011 & 0,9155 \\
\hline AB & $5,744 \mathrm{E}+06$ & 1 & $5,744 \mathrm{E}+06$ & 7,85 & 0,0088 \\
\hline AC & $2,069 \mathrm{E}+07$ & 1 & $2,069 \mathrm{E}+07$ & 28,28 & $<0,0001$ \\
\hline BC & $4,172 \mathrm{E}+05$ & 1 & $4,172 \mathrm{E}+05$ & 0,65 & 0,4283 \\
\hline A $^{2}$ & $3,454 \mathrm{E}+06$ & 1 & $3,454 \mathrm{E}+06$ & 5,35 & 0,0283 \\
\hline Residual & $1,808 \mathrm{E}+07$ & 28 & $6,458 \mathrm{E}+05$ & & \\
\hline Falta de ajuste & $1,669 \mathrm{E}+07$ & 1 & $1,669 \mathrm{E}+07$ & 323,56 & $<0,0001$ \\
\hline Error puro & $1,393 \mathrm{E}+06$ & 27 & 51582,3 & & \\
\hline Total & $1,054 \mathrm{E}+08$ & 35 & & & \\
\hline
\end{tabular}

Fuente: Software Design Expert 
Tabla 11. Análisis Estadística Descriptiva para los Datos del modelo 2FI

Tabla 11. Descriptive Statistics Data Analysis for the 2FI model

\begin{tabular}{|l|l|}
\hline Desviación Estándar & 803,62 \\
\hline Media & 5014.44 \\
\hline C.V. & 16,03 \\
\hline PRESS & $2,965 \mathrm{E}+007$ \\
\hline $\mathbf{R}^{2}$ & 0.8285 \\
\hline $\mathbf{R}^{2}$ Ajustado & 0.7856 \\
\hline $\mathbf{R}^{2}$ Predicho & 0,7188 \\
\hline Precisión Adecuada & 13,176 \\
\hline
\end{tabular}

Fuente: Software Design Expert
El valor Predicho de $\mathrm{R}^{2}$ de 0.7188 es aproximadamente igual al valor Ajustado de $\mathrm{R}^{2}$ de 0.7856 . El valor de la precisión Adecuada de 13,176, mayor de 4, significa que el modelo es adecuado para el diseño (Ver Tabla 11).

La siguiente formula muestra la rugosidad en la superficie del acero AISI 4140 como una función de las RPM, del Avance y de la Profundidad de corte. En este caso, la representación de la regresión para el modelo refinado en término de los factores codificados es:

Rugosidad $=5890,5+365.69^{*} A+1284.06 * B+16.19 * C-$
$423.69^{*} A^{*} B+804.19^{*} A^{*} C-114,19 * B * C-985,56^{*} A 2$

Esta ecuación sirve para predecir la rugosidad en la superficie del acero AISI 4140 o para determinar los niveles de los factores que minimicen la rugosidad. La ecuación final con las condiciones de los factores actuales es:

Figura 7. Gráfica de Contorno para la Rugosidad. a) Factor (A-B), b) Factor (A-C), y c) Factor (B-C)

Figure 7. Graphic contour for roughness. a) Factor (AB), b) Factor (AC), and c) Factor (BC)

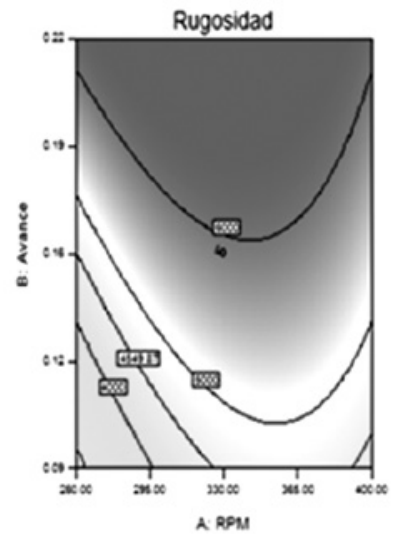

a

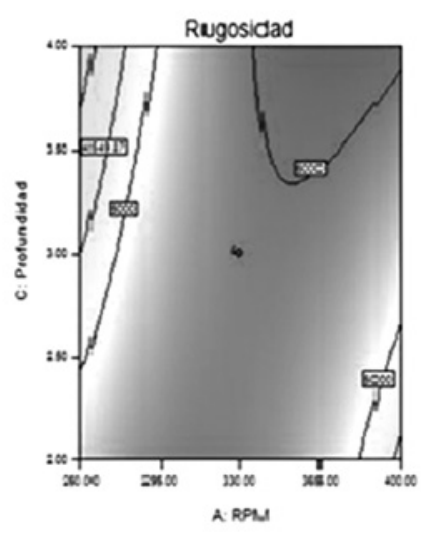

b

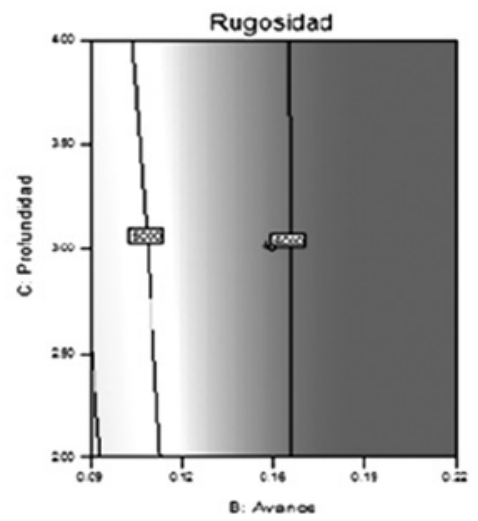

C

Fuente: Propia

Figura 8. Grafica de Superficie de Respuesta para la Rugosidad. a) Factor (A-B), b) Factor (A-C), y c) Factor (B-C) Figure 8. Grafica Response Surface Roughness. a) Factor (AB), b) Factor (AC), and c) Factor (BC

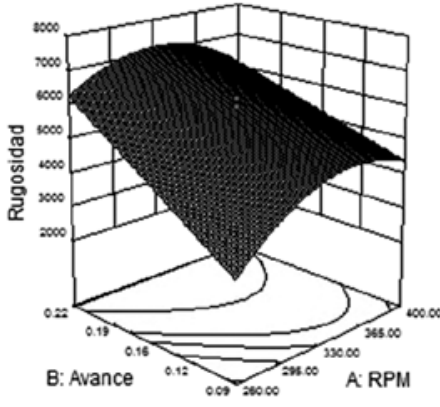

a

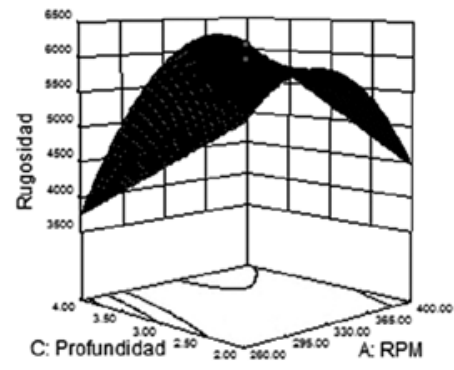

b

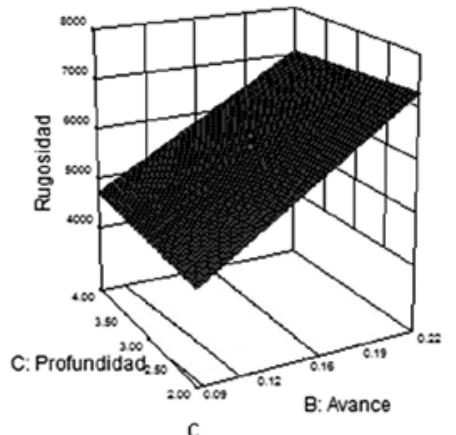

C

Fuente: Propia 


\section{Rugosidad $=+15054,00010+117,94147^{*} \mathrm{RPM}+$ $55753,98352 *$ Avance $-3502,68887^{*}$ Profundidad - 93.11813*RPM*Avance + 11.48839*RPM*Profundidad - 1756,73077*Avance*Profundidad - 0,20114*RPM ${ }^{2}$}

La gráfica de contorno y la gráfica de la superficie de respuesta tridimensional para la respuesta de la rugosidad son generadas por la ecuación de la rugosidad. La superficie de respuesta tridimensional proporciona esencialmente la misma información pero da una perspectiva distinta y más útil.

\section{CONCLUSIONES Y RECOMENDACIONES}

- Las revoluciones que entrega el torno (RPM) y el Avance de la herramienta respecto a la pieza demuestran ser dos factores significantes para la determinación de la calidad de la superficie de los modelos. De estos dos factores el Avance es el más influyente.

- El modelo satisface la normalidad, la independencia y las suposiciones de la variación constantes, por lo tanto, se considera eficaz en la solución de problemas de optimización de la rugosidad superficial para aceros 4140 sometidos a un proceso de cilindrado.

- El análisis estadístico de los datos indica claramente que el factor Avance (B) de la herramienta es más significativo que el factor RPM (A), revoluciones entregadas por el torno.

- La interacción RPM - Profundidad de corte $\left(\mathrm{A}^{*} \mathrm{~B}\right)$ también es bastante significante.

- La Profundidad de corte, factor C, no tiene mucha influencia en la rugosidad superficial.

- A pesar de que en los últimos años se ha encontrado mucha investigación en este campo, acompañada de muy buenos resultados, es importante concluir que el tema debe aún ser estudiado más profundamente, pues existen procesos donde la rugosidad es un factor muy importante (por ejemplo el fresado de paredes delgadas), y se hace necesario controlar las vibraciones para mejorar la rugosidad superficial y la tolerancia dimensional.

\section{REFERENCIAS}

[1] BENARDOS, P.G., VOSNIAKOS, G.C., (2003) Predicting surface roughness in machining: a review. International Journal of Machine Tools \& Manufacture, pp. 833-844

[2] Boothroyd G., Knight W.A. Fundamentals of Machining and Machine Tools, ${ }^{\text {nd }}$ ed. Marcel Dekker, 1989.

[3] Abouelatta, O, Madl, J. Surface Roughness prediction based on cutting parameters and tool vibrations in turning operations. Journal of Materials Processing Technology 118 (2001)269-277.
[4] Davim, J. A note on the determination of optimal cutting conditions for surface finish obtained in turning using design of experiments. Journal of materials processing technology 116(2001) 305-308

[5] Ghanij.A, Choudhuryi A, Hassanh H. Application of Taguchi method in the optimization of end milling parameters. Journal of Materials Processing Technology 145 (2004) 84-92.

[6] Correa M., et al. Factores que afectan el acabado superficial en los procesos de mecanizado: Técnicas de análisis y modelos. XXV Jornadas de Automática, 2004.

[7] Vivancos J, Ortiz J.A, Costa L. Luis C.J. Determining factors in the study of the surface roughness in the high-speed milling of hardened mould steel. 8th International Research/Expert Conference "Trends in the Development of Machinery and Associated Technology", TMT 2004, 15-18.

[8] Vivancos J, Luis C.J, Costa L, Ortiz J.A. Optimal machining parameters selection in high speed milling of hardened steels for injection moulds. Journal of Materiales Processing Technology 155-156 (2004) 1505-1512

[9] Vivancos J, Luis C.J, Ortiz J.A, Gonzalez H.A. Analysis of factors affecting the high speed side milling of hardened die steels. Journal of Materials Processing Technology, v 162163, 15 May 2005, 696-701.

[10] [ISO4287 1998], Norma UNE-EN ISO 4287: 1998. Especificación geométrica de productos (GPS), Calidad superficial: Método del perfil. Términos, definiciones y parámetros del estado superficial. Ed. AENOR 1999

[11] [ISO4288 1998] Norma ISO 4288: 1996. Geometrical Product Specification (GPS)-Surface texture: Profile method-Rules and procedures for the assessment of surface texture. Ed. ISO Standards, 1996

[12] [ISO 8785 1998] Standard ISO 8785: 1998. Geometrical Product Specification (GPS)-Surface imperfections-Terms, definitions and parameters. Ed. ISO Standards,1998

[13] Mongomery, D.C. Diseño y Análisis de Experimentos. Limusa Wiley, Mexico, 2003.

[14] Morera Steve. (2011) blogspot "Rugosidad, definición y características" Disponible desde <http://stevemorera. blogspot.com/2011/07/rugosidad.html > [Acceso 16 de junio de 2013]

[15] Briceo Mónica .(2011) blogspot. Ingeniería Electromecánica. Rugosidad superficial. Disponible desde <http:// monibriceo.blogspot.com/2011/07/rugosidad-superficial. html $>$ [acceso 20 de junio de 2013]

[16] Vallina Manuel (2013) blogspot. Mecanizado Básico. Variables del mecanizado. Disponible desde < http://manuelvallina.blogspot.com/2013_11_01_archive.html > [acceso 5 de noviembre de 2013] 